\title{
SPACE SIMULACRUM: A PARADOX OF CONCEPTUAL NOVELTY
}

\author{
HAZIRAH HANI HAMZAH, SYED SOBRI SYED ZUBIR, FAIRUZ REZA RAZALI, \\ ZAHARAH YAHYA \& FAIRUS KHOLID \\ Centre of Studies for Architecture, Faculty of Architecture, Planning \& Surveying, \\ Universiti Teknologi MARA, Malaysia
}

\begin{abstract}
The idea of public space, as it has developed in modern times, was space for the free use and enjoyment of the citizenry. The ambiance and character of public space should be determined not by any private or public authority, but by the ways in which people choose to use it. However, in Ho Chi Minh City, to be precise, political interventions to the existing local by-laws has restricted free access to public space and are eradicating the diversity of civic life. This is a new alliance of a business and state elite, set against civil society. What turns out to be 'messy' is the social life itself skateboarders, protesters, buskers, leafleteers, children playing games - that is, any activity that is not shopping or getting from A to B. The Space Simulacrum is intended mainly to contest against such restrictions, and to create points of diversion around the pedestrian boulevard, pulling the crowds away from the boulevard. The urban design scheme manipulates the use of space and time, combined with hypothetical injections of programs, in order to act as socio-cultural catalysts to increase pedestrian traffic in Downtown District 1, Ho Chi Minh City, during the daytime by creating an altered reality, in which perceptions and experiences or space, duration and gravity are transformed. The methodology and strategy of plugging in social activities on some potential areas, as well as constructing minor installations throughout the site, are intended to haul people into some sort of black holes, and making them forget about the passing of times. These interventions are hopefully able to make a comprehensive rediscovery of the idea of public space, and to remediate the public realm in the immediate vicinity of the Nguyen Hue Pedestrian Boulevard.
\end{abstract}

Keywords: space, time, space-time, diversion, altered reality, public space, public realm, pedestrian.

\section{INTRODUCTION}

Ho Chi Minh City (HCMC) is Vietnam at its most dizzying: a high-octane city of commerce and culture that has driven the country forward with its pulsating energy. A chaotic whirl, the city breathes life and vitality into all who settle here, and visitors cannot help but be hauled along for the ride - it is a city of energy and discovery. Wander through timeless alleys to incense-infused temples before negotiating chic designer malls beneath sleek 21st-century skyscrapers. The ghosts of the past live on in buildings that one generation ago witnessed a city in turmoil, but now the real beauty of the former Saigon's urban collage is the seamless blending of these two worlds into one exciting mass, creating a dynamic urban sphere in very special sense. The downtown area, still referred to as Saigon, is growing at a rate that will equal Tokyo by 2020. If Hanoi is the political capital of Vietnam, Ho Chi Minh City is the commercial heart and a magnet for foreign investors. Progress aside, Saigon is steeped in tradition, a living monument to the past that wears its culture proudly on its sleeve. Decorated with colonial and French style architecture throughout the city, Saigon houses a unique mixture of past and present.

The proposed urban design intervention site is located in the Central Business District, CBD of Ho Chi Minh City, sets in District 1, one of 19 urban districts (quận) in the city. 


\section{SITE SETTING}

District 1 is the financial and commercial hub of Ho Chi Minh City. Most travelers will spend the majority of their time here. Home to most of the major sights and an expanding number of bars and restaurants, District 1 was designed by French architects during the colonial era, which explains the wide, tree-lined boulevards and sporadic locations of parklands. The urban intervention site is situated at the center of the heart of District 1 , within the visual locality of the Saigon Opera House and Committee Hall - approximately 23 acres of site parameters; Fig. 1(a). It is the busiest district in the city with the highest living standards. Dong Khoi street and Nguyen Hue boulevard in the district are the city's two main commercial centers, besides other major roads such as Le Loi Street and Hai Ba Trung Street, as shown in Figs 1(b) and 1(c).

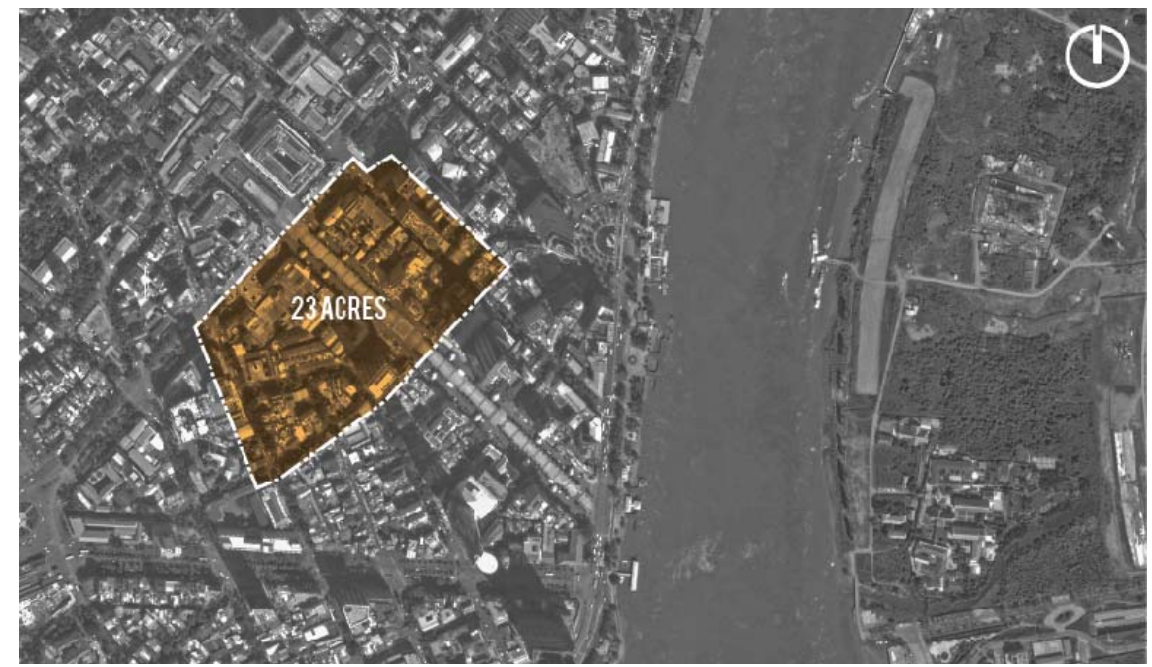

(a)

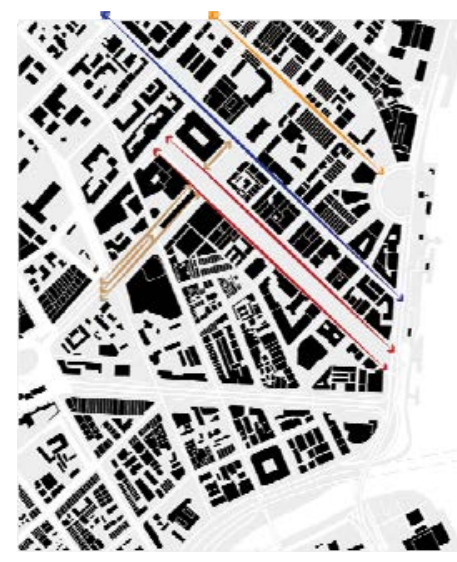

(b)

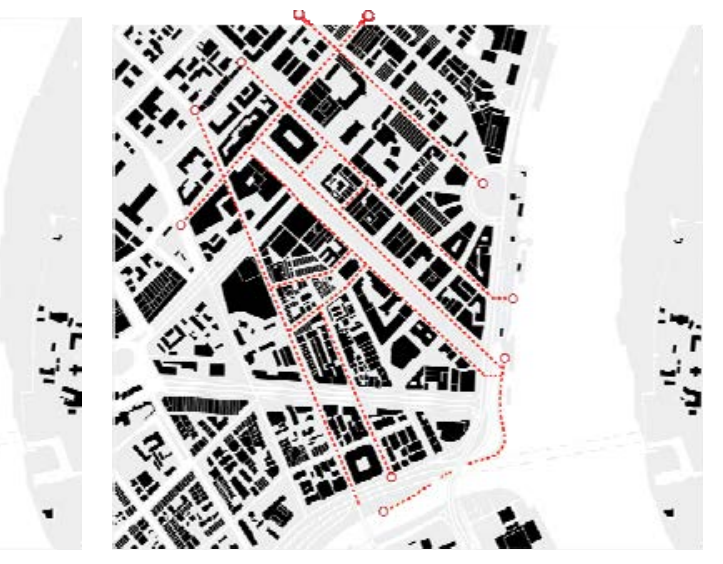

(c)

Figure 1: (a) Site parameter of the urban design intervention; (b) Major roads in Downtown District 1; (c) Major pedestrian linkages. 


\section{POTENTIALS AND DILEMMAS}

\subsection{Site potentials}

The proposed site has a lot of potentials, and can be broken down into few main ones - land use and land budget, accessibility, imageability and area identity. Nguyen Hue Street, and its neighbouring street particularly Le Loi Street and Dong Khoi Street, have prime land value, approximately VND162 million (roughly US\$7,600) per $\mathrm{m}^{2}-$ the most expensive land value in Ho Chi Minh City and the whole of Vietnam. The proposed site will most likely to emerge a denser, more compact city. The fact that the area is filled with commercial-dominated buildings, with future subway line and station that can generate a bigger volume of people and businesses should be used to its advantages. Strong horizontal axis links major landmarks towards the waterfront, coupled with presence of power brands in multiple locations are identified as nodes that draw people to the area. Fig. 2(a) summarized few potentials of the site that are taken into considerations to generate alternative urban design intervention schemes.

\subsection{Site dilemmas}

Downtown District 1, particularly in the vicinity of the proposed site, although is full of potentials, have its own dilemmas. Interestingly, the prominent pedestrian boulevard in Nguyen Hue Street, which is the main public space provided by the City Council, is seemingly hot due to lack of shaded area, insufficient trees along the boulevard and the sidewalks of the neighbouring street. Besides that, lots of restrictions are imposed especially along the sidewalks, boulevard and other public areas. Street vendors are also banned from using the sidewalks facing the boulevard. Issues regarding this pedestrian boulevard are what triggered the project's thirst for urban intervention in this paper. Other dilemmas of the site are explained in Fig. 2(b).

\section{BACKGROUND THEORY: THE SPACE-TIME ODYSSEY}

\subsection{Conceptions of space}

Space, is defined as a continuous area or expanse that is free, available, or unoccupied. However, when we talk about space in relations to architecture, it is the infinity which surrounds us, and of which only lines can make us aware. Space, as we see it, is like the interplay between solid matter and void. In the early and mid-20th century, the concept of space was critical in defining the modern movement in architecture. Notions of architectural space related to the coherence between the interior and exterior of buildings emerged as a new feature [1]. Counteracting previous understandings of architecture as a progression of styles, space became a privileged quest in the architectural domain. Space is first conceived of as a property of the mind and then realized as physical matter. In the move from the metaphysical concept to the physical reality, the word 'space' has to cover a whole variety of conditions, and it is here that confusion arises between concept and reality. Space is a continuous area or expense that is free, available, or unoccupied. It is the infinity which surrounds us, and of which only lines can make us aware. 

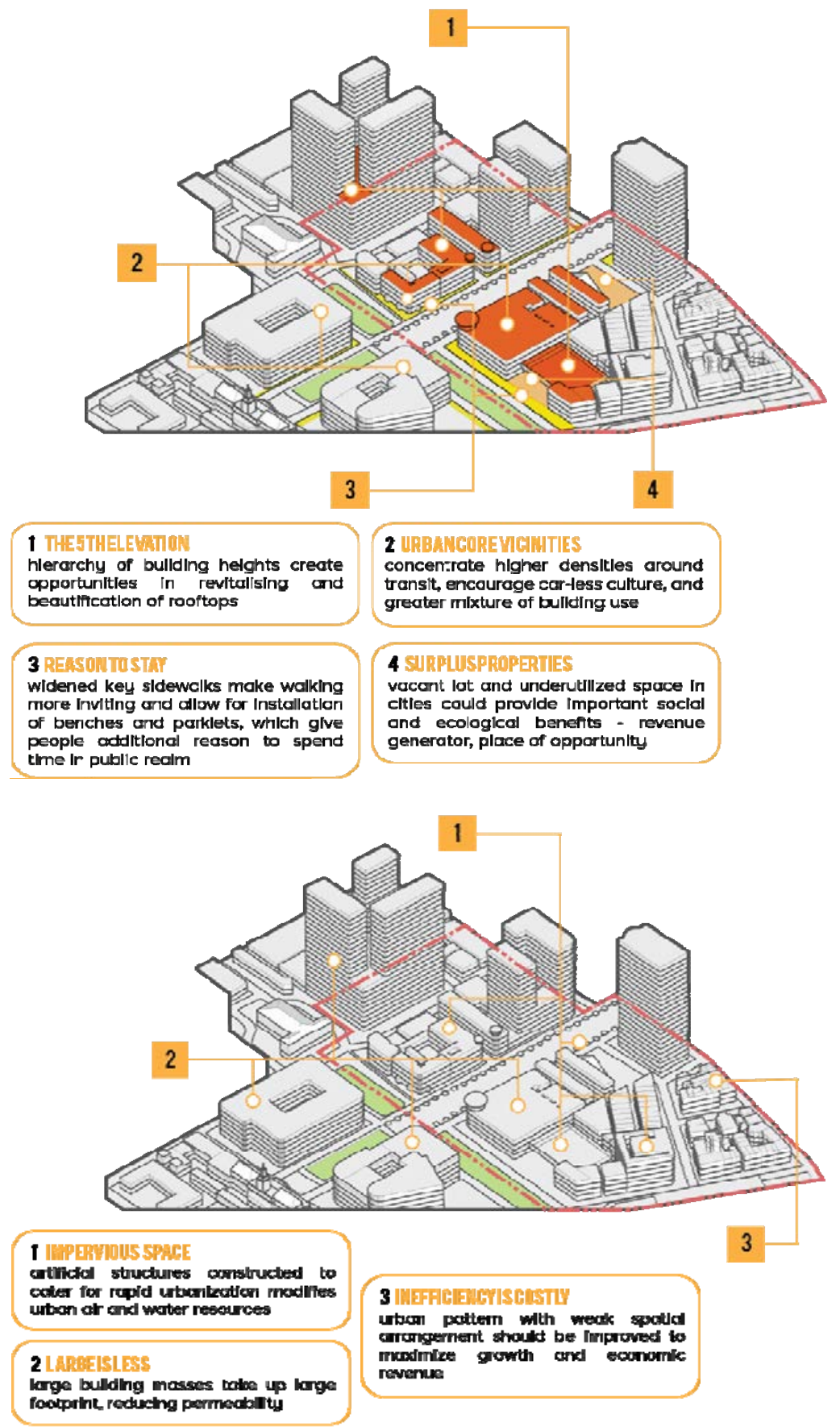

Figure 2: (a) Site potentials: diagram and summary; (b) Site dilemma: diagram and summary. 


\subsection{Conceptions of time}

Time is the most mysterious dimension of the physical reality and human consciousness. It appears self-evident in the context of everyday life, but beyond comprehension in deeper scientific and philosophical analyses. The concept of time is an important and vital issue in architecture and urban planning. Time means simply the perception of change in one place. However, to talk about the future means to understand time and it is important to understand that time is not such a simple or obvious phenomenon, but that one has to exercise considerable ingenuity to fit it into a coherent picture of the universe. Time also gives us a place like feeling to it suggesting that time is a dimension.

\subsection{From space and time, to space-time}

Space and time are doomed to fade away into mere shadows, and only a kind of union of the two will preserve an independent reality. As such that time is relative; it can vary for different observers depending on the speed through space. To Einstein, time is the "fourth dimension." On the other hand, space is described as a three-dimensional arena, which provides a traveller with coordinates such as length, width and height; basically, showing location [2]. Time provides another coordinate and direction, although conventionally, it only moves forward.

\subsection{Time-space compression}

Time-Space compression refers to any phenomenon that alters the qualities of and relationship between space and time. The constantly increasing speed of change tends to reduce the importance of any certain change [3]. The gist of this concept is that all space can be divided into either smooth spaces (where the occupant has limited to no interaction with the exterior of the space and cannot accurately perceive changes in distance and time) and striated spaces (where the occupant can easily measure changes in distance and time due to familiar understanding of the exterior).

\subsection{Time-free perception}

Regardless of its ephemeral and mystical nature, time is the crucial fluid of our mental lives. We do not live in a fixed, objective reality; we live in a mental reality that perpetually keeps flowing back and forth between reality, dream and imagination. Our mental reality has no fixed boundaries, no fixed temporal order or given categories. The idea of a "time-free" consciousness, or mode of perception, was once inferred as a necessary condition for creative thought [4]. Time-free association, remembrance and dreaming, in fact, also seem to be the condition of our normal daydreaming as well as concentrated thought; in these mental states, we detach ourselves from the progression of time.

\subsection{Zeitgeist}

From the German zeit, time and geist, ghost, and translated into English as the 'spirit of the times', a prevailing theory that architecture cannot possibly be a leading factor of human culture rather it should only strive to reflect the times. Zeitgeist is the general cultural, intellectual, ethical, spiritual, or political climate within a nation or even specific groups, along with the general ambiance, morals, sociocultural direction, and mood associated with 
the current era - anxiety, aggression and collision. Expressing the concept of the sense or rationality in order of things and the succession of one state of affairs after another according to some kind of lawful process, translates what zeitgeist is [5]. Zeitgeist does not indicate a homogenous state of affairs in which everyone goes along with the same idea, but rather expresses the fact that in any given society, there is a certain 'language', culture, or range of concepts in which every dispute, every contradiction must be fought out.

Hegel's Triad of Thesis, Antithesis, and Synthesis (Fig. 3) can be deduced as a character is in its comfort zone but wanted something, and willing to enter an unfamiliar situation, adapt to it, to get what they wanted by paying a heavy price for it. Then, return to their familiar situation and having things changed.

\section{DESIGN STATEMENT}

The main intentions of this urban design escapade is to reconstruct a socio-cultural catalyst to increase pedestrian traffic in Downtown District 1, Ho Chi Minh City during daytime by creating an altered reality, in which perceptions and experiences of space, duration and time are transformed. Remediation of the public realm in vicinity of the pedestrian boulevard can be achieved by providing the downtown quality open space in sufficient quantity and variety to meet the needs of downtown workers, residents and visitors, at the same time protesting/countering authority's restrictions on do's and don'ts in public space, specifically the pedestrian boulevard and sidewalks, as shown in Fig. 4.

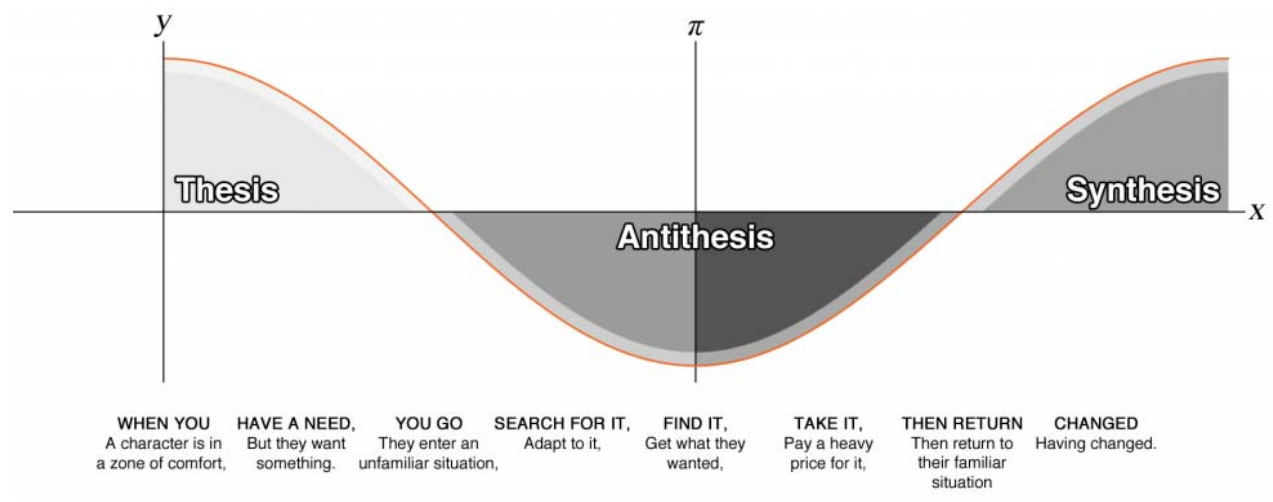

Figure 3: Hegel's Triad of Thesis, Antithesis and Synthesis.

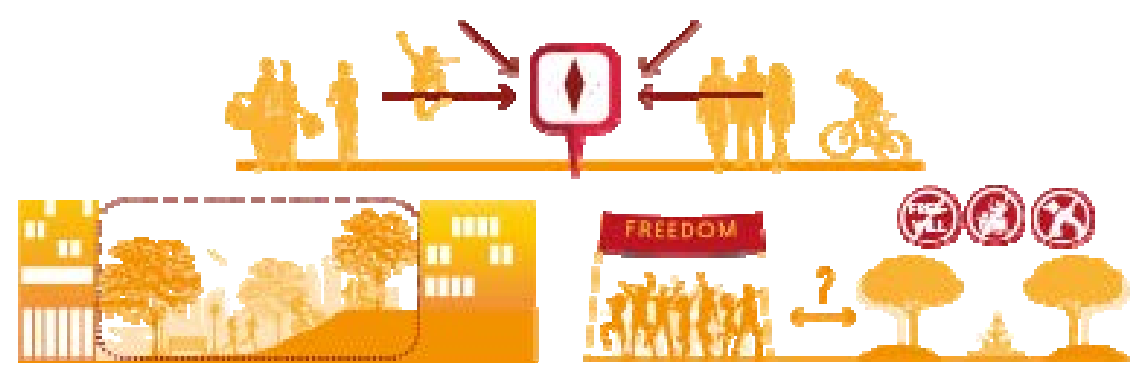

Figure 4: Graphical interpretation of intended design intervention. 
By remediating the public realm through architectural protest, this scheme is meant to confront the authority with issues raised on the usage of the space. The main issues include: restrictions imposed, lack of amenities, and the financial spending in construction of the MRT project. The project explored different visual and physical approaches to inform the public of the posed issues while analyzing how design can serve as a tool to shock, instigate and intervene in the community. The objective is to incite ideas that can potentially be turned into actions for a better public realm.

\section{DESIGN INTERVENTION: SPACE SIMULACRUM}

A simulacrum is more than just a useless image; it is a deviation and perversion of imitation itself - a false likeness. Therefore, the term 'Space Simulacrum' used repetitively in this paper is essentially physical spaces defined as static, deceiving its viewer on the level of experience, a manipulation of the senses which transforms the unrealistic into the believable. Space Simulacrum is a manifesto of architectural protest to confront the authority with issues raised on the usage of public realm. The idea of Space Simulacrum explored different visual and physical approaches to inform the public of the posed issues while analyzing how design can serve as a tool to stimulate, instigate or intervene in the community. Fig. 5 depicts a visual impression of the intended scheme of the project.

\subsection{Design concept}

6.1.1 Black holes: doorway to an alternate reality

Black holes are incredibly massive, but cover only a small region. Theoretically, to make a black hole, there must be a presence of 'mass' that distorts the local 'space-time' as if it were a rubber sheet. Coming back to interpreting this concept into design theory, in order to create a black hole or a crowd-puller, hypothetical injection of activities must exist. The analogies of this concept are depicted in Figs 6(a) and 6(b).

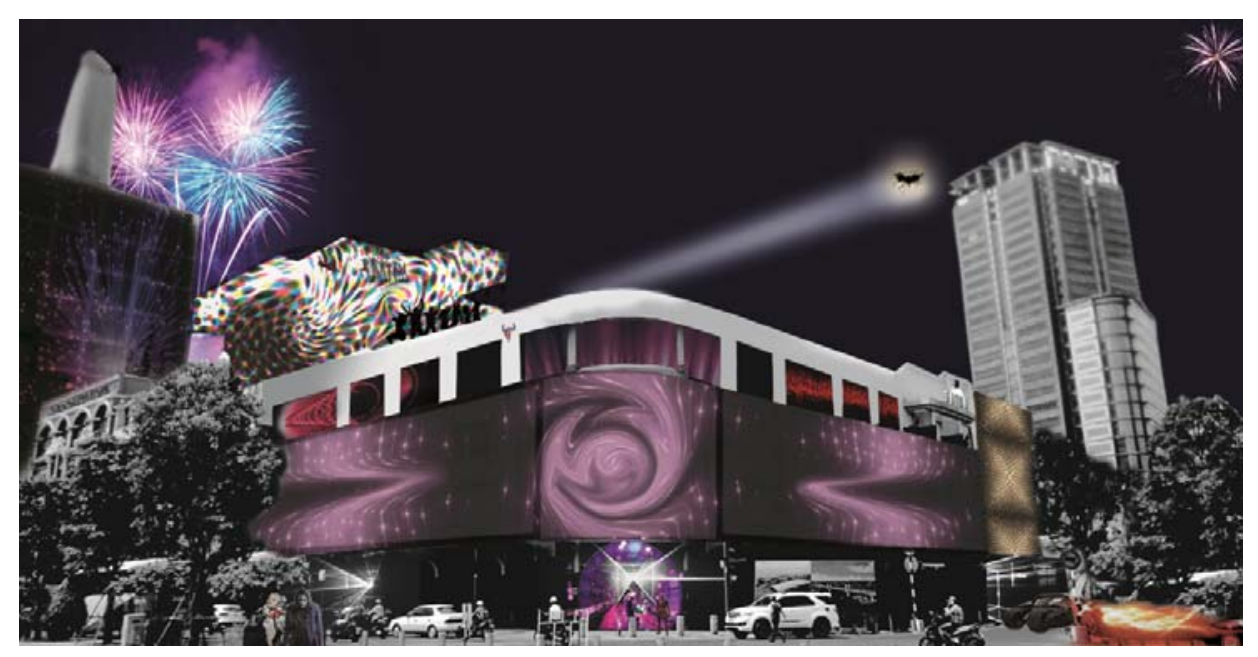

Figure 5: Visual impression of a space simulacrum. 

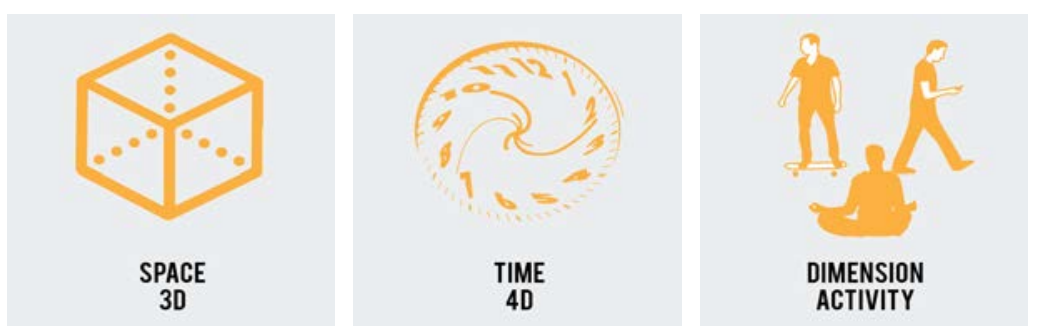

(a)

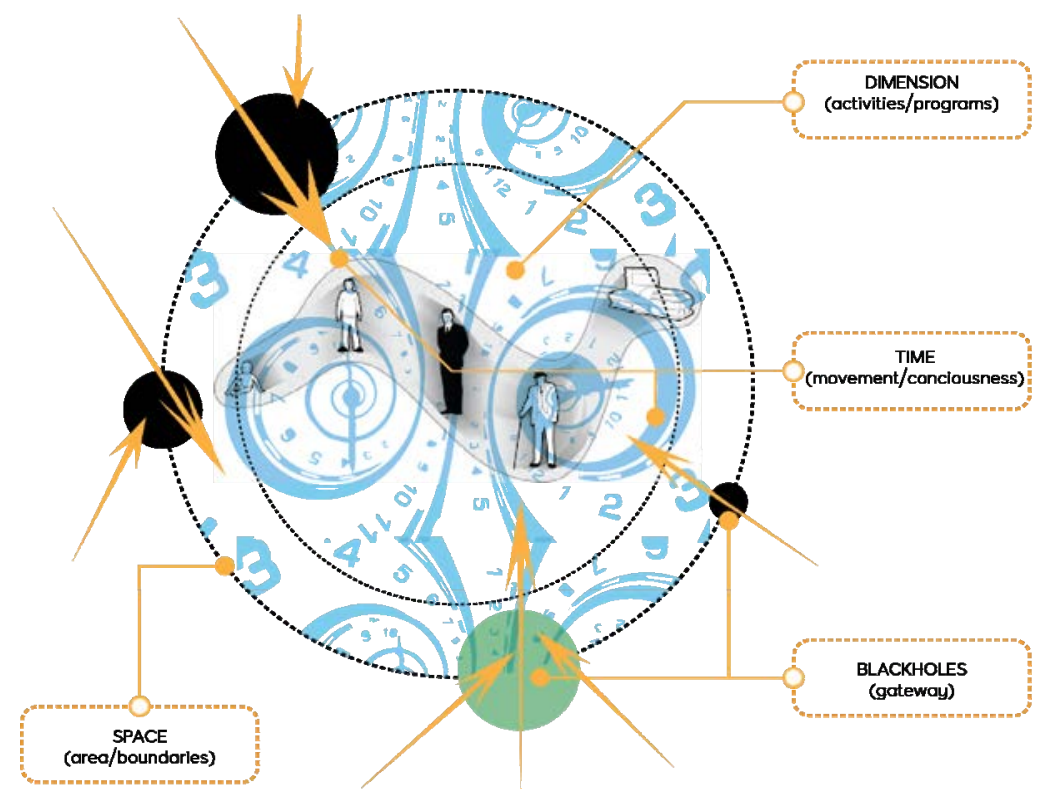

(b)
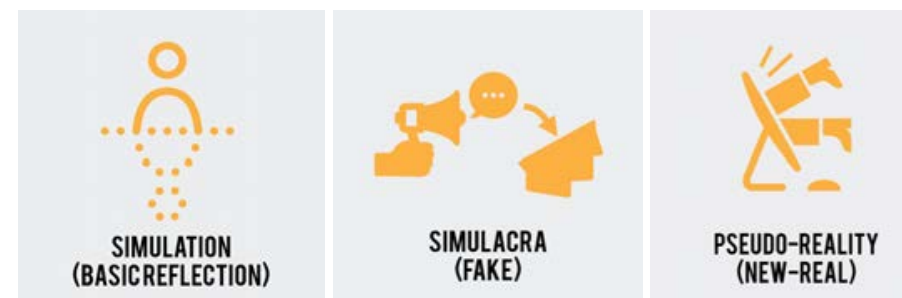

(c)

Figure 6: (a) Analogies of a black hole, inferred into design keywords; (b) Conceptual ideas deduced into graphical diagram; (c) Analogies of a hyperreality.

\subsubsection{Hyperreality}

A term associated with the effects of mass culture reproduction, suggesting that an object, event, and experience so reproduced replaces or is preferred to its original: that the copy is 'more real than real'. Hyperreality is associated especially with cultural tendencies and a 
prevailing sensibility in contemporary American society [6]. Hyperreality is a special kind of social reality in which a reality is created or simulated from models, or defined by reference to models - a reality generated from ideas. The term has implications of 'too much reality' - everything being on the surface, without mystery; 'more real than reality' - too perfect and schematic to be true, like special effects; and 'para-reality', an extra layer laid over, or instead of, reality. It is experienced as more real than the real, because of its effect of breaking down the boundary between real and imaginary. In semiotics and postmodernism, hyperreality is an inability of consciousness to distinguish reality from a simulation of reality. There is no reality in these places, only a construct that is designed to represent reality, allowing the person to exist temporarily in a world outside of what is real.

In deducing and reinterpreting Hyperreality in this scheme, it can be concluded that Hyperreal surroundings can be created through the 4 steps explained below:

a) Constructing undistinguishable reality from simulation of reality

b) Fiction + reality blended together

c) Co-mingling of physical + virtual reality

d) Manipulation of people's perception of space and time

\subsection{Strategy: confrontations, points of diversions and distractions, stimuli}

As nouns the difference between distraction and diversion is that distraction is something that distracts while diversion is a tactic used to draw attention away from the real threat or action. The main strategy used in this project is meant to do just this. Subsequent strategies derived from the black hole and hyperreality concepts are the establishment of physical and visual stimuli that draw people away from the pedestrian boulevard, and drawing them in to the areas and spaces adjacent to the boulevard, a mere sign of protesting and opposing.

\subsection{Hypothetical space programs}

\subsubsection{Social dimension}

The idea of a social dimension is that the program is intended to trigger social interaction man to man interaction, man to object interaction, man and buildings interaction. Social dimension, as depicted in Fig. 7(a), aims to attract people of diverse backgrounds and experience, to make understanding and appreciating one another's differences, as well as to make people being respectful of those different from them.

\subsubsection{Cultural dimension}

Cultural dimension aims to activate cultural-based activities, at the same time aids in expanding the knowledge and awareness of the local culture. In this project, the cultural dimension is strategically constructed nearby the Saigon Opera House, in which has the most potentials to draw people to the area after a play/show, for example. The notion of this 'cultural dimension' is shown in Fig. 7(b).

\subsubsection{Creative dimension}

The creative dimension is where the brains of the artistic and imaginative collide. The idea of a creative dimension (Fig. 7(c)) is to invoke sense of partnership with philanthropic, private organizations and educational institutions to provide a broad range of services around space for arts and cultural production. The program brokers, lets, sublets and develops affordable space for the creative industries. 


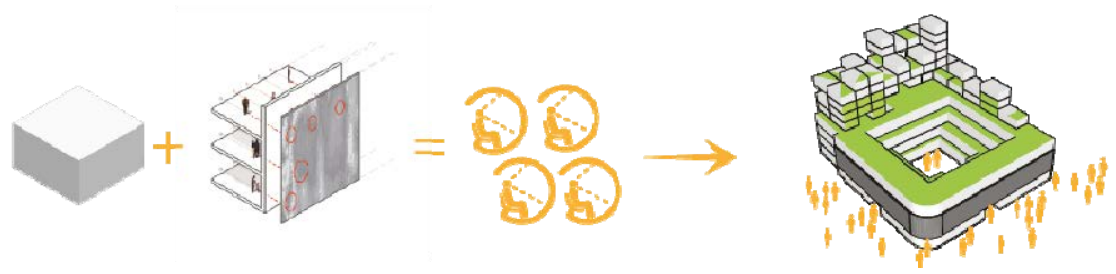

(a)

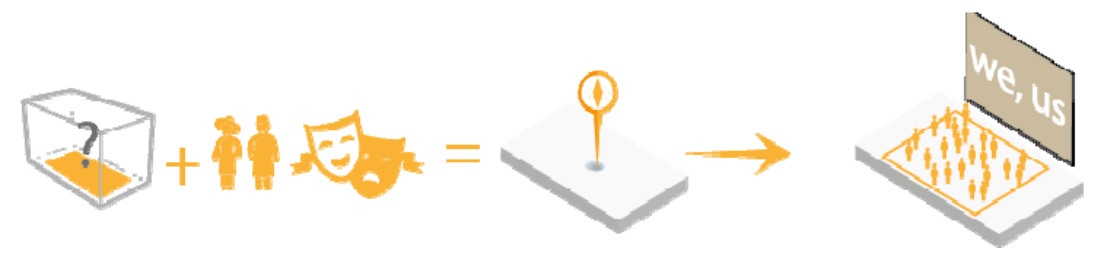

(b)

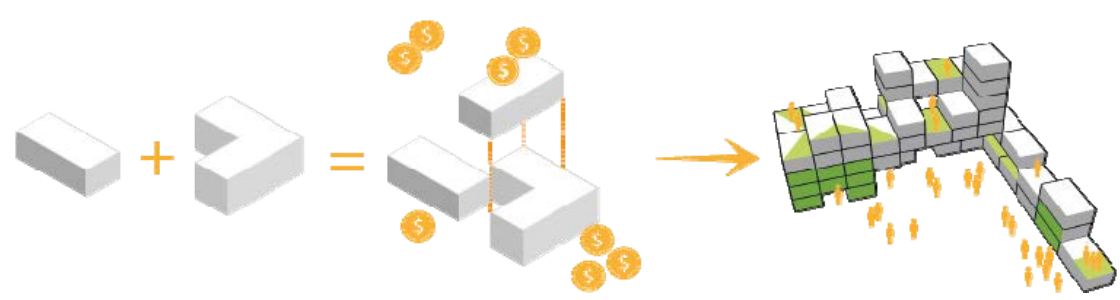

(c)

Figure 7: (a) Notion of a social dimension; (b) Notion of a cultural dimension; (c) Notion of a creative dimension.

\section{CONCLUSION}

One would expect that in the city many places exist where citizens with all different backgrounds can meet, without exceptions: namely the public space. However, the fact that the search for alternatives keeps emerging, raises some questions about our contemporary public space. The notion of 'public space' is difficult to define. Of the same order as the impossibility of rediscovering an absolute level of the real, is the impossibility of staging an illusion. Illusion is no longer possible, because the real is no longer possible. Therefore, the tendency of Space Simulacrum is towards achieving this virtue in space and time.

\section{ACKNOWLEDGEMENT}

We would also like to extend our utmost appreciation to the Faculty of Architecture, Planning and Surveying, UiTM in providing a grant for the realization of this paper.

\section{REFERENCES}

[1] Moma.org, Conceptions of space: recent acquisitions in contemporary architecture, Online, 2014. http://www.moma.org. Accessed on: 23 Jun. 2016.

[2] Hawking, S., Space and time. A Brief History of Time, ed. S. Hawking, 1st ed., Bantam Books: New York, pp. 15-37, 1998. 
[3] Harley, D., The experience of space and time. The Condition of Postmodernity: An Enquiry into the Origins of Cultural Change, ed. D. Harley, 1st ed., Wiley-Blackwell: Oxford, pp. 260-284, 1991.

[4] Ehrenzweig, A., The Psychoanalysis of Artistic Vision and Hearing: An Introduction to a Theory of Unconscious Perception, 1st ed., Sheldon Press: London, 1975.

[5] Marxists.org, Marxism: The spirit of time - zeitgeist, Online, 1999. http://www.marxists.org/. Accessed on 19 May 2016.

[6] Ceasefiremagazine.co.uk, An A to Z of theory - Jean Baudrillard: Hyperreality and implosion, Online, 2012. https://ceasefiremagazine.co.uk/in-theory-baudrillard-9/. Accessed on 20 May 2016. 